\title{
MANEJO DE LA NUTRICIÓN DEL CULTIVO DE CACAO EN LA REGIÓN DE SANTO DOMINGO - ETAPA DE ESTABLECIMIENTO DEL HUERTO
}

\author{
Autores: \\ Miriam Recalde ${ }^{1}$ \\ Manuel Carrillo \\ José Sánchez ${ }^{1}$ \\ Rosa Moreno'
}

1. M. Recalde, J. Sánchez, R. Moreno

Universidad Tecnológica Equinoccial, Extensión Santo Domingo, km 4 1⁄2 Vía a Chone,

Santo Domingo de los Tsáchilas - Ecuador

mrecalde@ute.edu.ec

2. M. Carrillo

INIAP, Estación Experimental Litoral del Sur “Dr. Enrique Ampuero”

REVISTA DE INVESTIGACIÓN CIENTÍFICA 



\section{Resumen}

Se diseñó un estudio a largo plazo para evaluar el efecto del manejo nutricional sobre el comportamiento morfoproductivo de dos cultivares de cacao (Theobroma cacao) desde la siembra hasta cuando el huerto estabiliza la producción. El estudio se inició en marzo del año 2009, en este artículo se reportan los datos obtenidos en dos años de establecimiento de la huerta experimental. El sitio está ubicado en la Granja Experimental de la Universidad Tecnológica Equinoccial, Extensión Santo Domingo. Se sembraron 1130 plantas de cacao a un distanciamiento de 3,5 x 3,5 m. Se evaluaron dos tipos de material genético (cacao Nacional y el clon CCN51) con siete tratamientos de fertilización (testigo, N, NP, NPK, NPKSMg + Micros, Gallinaza y NPKSMg + micros + Gallinaza). Para el análisis estadístico se utilizó un diseño de bloques al azar con arreglo factorial ( 2 × 7). Las variables morfométricas evaluadas en el periodo de establecimiento fueron diámetro del tallo y altura de planta y las variables productivas, índice de vigor, incidencia de escoba de bruja (moniliophtora perniciosa) y rendimiento. Se observaron diferencias $(\mathrm{P}<0.05)$ entre cultivares en altura de planta e índice de vigor ratificando la hipótesis de un mayor crecimiento vegetativo del cacao Nacional frente al $C$ CN51. No hubo diferencia ( $>>0.05)$ de los tratamientos en la incidencia de escoba de bruja. Los rendimientos del cultivar $\mathrm{CCN}_{51}$ fueron mayores a los del cacao Nacional $(\mathrm{P}<0.05)$ a pesar de su menor tamaño, lo que sugiere que el sumidero de nutrientes y otros compuestos metabólicos del tronco de la planta se consolida tempranamente en el cultivar CCN51, condición que permitiría mayor acumulación de rendimiento el periodo de producción de la edad adulta de la plantación. El experimento continuará evaluándose en el campo por varios años.

Palabras clave: Cacao, Theobroma cacao, Moniliophtora perniciosa, fertilización. 


\section{Abstract}

A long term experiment was initiated to evaluate the effect of the nutritional management over the morphoproductive behavior of two cocoa (Theobroma cacao) cultivars from planting to production stabilization. The study started on March 2009 , and this article reports the results obtained during the two years period of the experimental orchard establishment. The site is located at the Experimental Farm of the Universidad Tecnológica Equinoccial, Campus Santo Domingo, Ecuador. A total of 1130 seedlings were planted 3.5 by 3.5 m apart. Two cocoa genetic materials (National and CCN51) with 7 fertilization treatments (control, N, NP, NPK, NPKSMg + Micros, Chicken manure y NPKSMg + Micros + Chicken manure) were valuated. For the statistical analysis a completely randomized block design with a factorial arrangement ( $2 \times 7)$ was utilized. The morphometric variables evaluated during the establishment period were stem diameter, and plant height and the productive variables were vigor index, incidence of witch's broom disease (Moniliophtora perniciosa) and yield. Differences among cocoa cultivars were observed $(\mathrm{P}<0.05)$ ratifying the hypothesis of a greater vegetative growth of the National ETT95 cultivar over the CCN51. No treatment differences were observed (P>0.05) for witch's broom. CCN51 cocoa yield was greater than National EET95 ( $P<0.05)$ on spite of the lower vegetative growth, suggesting that the nutrient and other metabolic compounds sink in the plant truck consolidates early in the CCN51 genetic material, condition which would promote greater yield during the adult life of the orchard. Evaluation of treatments in the experiment will continue for several years.

Key words: Cocoa, Theobroma cacao, Moniliophtora perniciosa, yield, fertilization. 


\section{Introducción}

Ecuador es el primer productor de cacao (Theobroma cacao $L$ ) fino de aroma del mundo, conocido también como cacao Nacional. En el país se cultiva además el clon CCN51 (Colección Castro Naranjal, cruce 51), material de alto rendimiento y buen contenido de grasas, pero de sabor y aroma inferior al cacao Nacional. A pesar de su menor calidad, el CCN51 mantiene un buen mercado y el área cultivada con este material crece constantemente (INIAP, 2009).

El cultivo de cacao es uno los principales generadores de divisas y fuentes de trabajo para la población rural de Ecuador. El III Censo Nacional Agropecuario del 2002 reportó que existían 433978 ha destinadas al cultivo de cacao distribuidas en 16 provincias. El Censo también determinó que solamente en 2294 ha se utilizaban fertilizantes y tan solo en 2272 aplicaban fungicidas (SICA, 2003). La superficie nacional cosechada con cacao en el año 2011 creció a 521091 ha (INEC, 2012) y se han producido cambios en el manejo, particularmente con la introducción de nuevas variedades de cacao Nacional y la reposición de huertos viejos. Sin embargo, la situación general con respecto al manejo del cultivo ha cambiado poco en los últimos años. Estas condiciones de manejo inadecuado del cultivo han hecho del propietario un simple cosechador y no un cultivador de cacao.

En la provincia de Santo Domingo de los Tsáchilas, los agricultores han venido sustituyendo la ganadería con cacao que ha cubierto una buena proporción de superficie total cultivada en la región (CORPEI, 2009). En esta zona se ha cultivado cacao Nacional desde hace mucho tiempo, en las mismas condiciones técnicas discutidas anteriormente para el resto del país. Sin embargo, en la actualidad se ha incrementado apreciablemente el cultivo de cacao CCN51 como una opción de producción.

Las labores de manejo como podas sanitarias y de formación, control de malezas, enfermedades e insectos y la nutrición pueden mejorar la productividad de los cultivares de cacao. Los cacaos nacionales tradicionales producen rendimientos bajos, pero los nuevos cultivares, como el EET95, tienen un mayor potencial de rendimiento (Amores, 2010), sin embargo, esto todavía no se ha podido documentar adecuadamente. Se diseñó un experimento de largo plazo para evaluar el efecto del manejo nutricional del huerto sobre el comportamiento morfo-productivo y sobre la incidencia a escoba de bruja (Moniliophthora perniciosa) del cacao Nacional y del clon $\mathrm{CCN}_{51}$ desde la siembra hasta cuando se estabiliza la producción. La hipótesis planteada es que la fertilización balanceada mejora las características fisiológicas y de tolerancia a enfermedades del cacao nacional y del clon $\mathrm{CCN}_{51}$, condiciones que inciden positivamente en el rendimiento y la rentabilidad del cultivo. Este artículo reporta los resultados de los dos primeros años de establecimiento del cultivo.

\section{Materiales y Métodos}

El periodo de evaluación reportado en este artículo corresponde a marzo del 2009 a febrero del 2011, periodo que comprende la etapa de establecimiento del cultivo. El experimento está ubicado en la Granja Experimental de la Escuela de Ingeniería Agropecuaria de la Universidad Tecnológica Equinoccial, Extensión Santo Domingo, ubicada en el km 4¹/2 vía Chone a 00¹3’ 50" de latitud Sur y $79^{\circ} 12^{\prime} 26^{\prime \prime}$ de longitud Oeste a una altitud de $552 \mathrm{msnm}$. Se están evaluado dos tipos de material genético (cacao tipo Nacional y CCN51) y 7 tratamientos de fertilización (Cuadro 1). Las variables fueron evaluadas utilizando un diseño de bloques al azar en arreglo factorial $(2 \times 7)$ con tres repeticiones. El modelo estadístico utilizado fue el siguiente:

$$
\mathrm{Y}_{\mathrm{ijk}}=\mu+\beta_{\mathrm{i}}+\tau_{\mathrm{i}}+\delta_{\mathrm{ij}}+\rho_{\mathrm{k}}+(\tau \rho)_{\mathrm{ik}}+\varepsilon_{\mathrm{ijk}}
$$

Donde: $\mathrm{Y}_{\mathrm{ijk}}=$ Variable de respuesta; $\mu$ = Media General; $\beta_{\mathrm{i}}=$ Efecto de Bloques; $\tau_{\mathrm{i}}=$ Efecto del j-ésimo genotipo; $\delta_{\mathrm{ij}}=$ Error asociado a las unidades experimentales; $\rho_{\mathrm{k}}$ = Efecto de la fertilización; $(\tau \rho)_{\mathrm{ik}}=$ Efecto de la interacción entre los genotipos y los niveles 
de fertilización y $\varepsilon_{\mathrm{ijk}}=$ Error Aleatorio. Se realizó una prueba de comparación de medias con Tukey con $\alpha=0.05$.

Las variables evaluadas en este periodo de la investigación fueron diámetro del tronco, altura de planta, índice de vigor, incidencia de escoba de bruja (Moniliophtora perniciosa) y rendimiento. La altura de planta se registró midiéndolo desde el suelo hasta el punto terminal de la rama más alta, el diámetro de tallo se midió a $15 \mathrm{~cm}$ de altura desde el suelo. El índice de vigor refleja el volumen de biomasa de la planta (m3) y se determinó midiendo la circunferencia del tallo en la porción más gruesa del injerto, la altura de planta y el diámetro de la corona de acuerdo a lo sugerido por (López, 2007):

$$
\text { Índice de vigor }(\text { IndVig })=\left(\frac{C^{2}}{4}\right)\left(H^{2}+\frac{D C}{4}\right)^{-2}
$$

donde $\mathrm{C}=$ circunferencia, $\mathrm{H}$ = altura de la planta y $\mathrm{DC}=$ diámetro de la copa

La incidencia de escoba de bruja se determinó contabilizando el número de escobas desarrolladas en los brotes terminales y las axilas de las ramas de una planta calculando luego el porcentaje presente en función del número de plantas por tratamiento. El rendimiento midió la producción de almendras durante el periodo de evaluación.

\begin{tabular}{|c|c|c|c|c|c|c|c|c|c|c|c|}
\hline \multirow[t]{3}{*}{ Genotipo } & \multirow[t]{3}{*}{ Fertilización } & \multicolumn{10}{|c|}{ 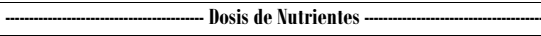 } \\
\hline & & $\mathrm{N}$ & $\mathrm{P}_{2} 0_{5}$ & $\mathrm{~K}_{2} \mathrm{O}$ & $\mathrm{s}$ & Mg0 & $\mathrm{N}$ & $\mathrm{P}_{2} 0_{5}$ & $\mathrm{~K}_{2} \mathrm{O}$ & $s$ & Mg0 \\
\hline & & \multicolumn{5}{|c|}{ g planta ${ }^{-1}$} & \multicolumn{5}{|c|}{$\mathrm{kg} \mathrm{ha}^{-1}$} \\
\hline \multirow{7}{*}{ Nacional } & Testigo & 0 & 0 & 0 & 0 & 0 & 0 & 0 & 0 & 0 & 0 \\
\hline & $\mathrm{N}$ & 70 & 0 & 0 & 0 & 0 & 57 & 0 & 0 & 0 & 0 \\
\hline & $\mathrm{NP}$ & 70 & 12 & 0 & 0 & 0 & 57 & 10 & 0 & 0 & 0 \\
\hline & NPK & 70 & 12 & 25 & 0 & 0 & 57 & 10 & 20 & 0 & 0 \\
\hline & NPKSUIg+Micros (Com)' $)^{1}$ & 70 & 12 & 25 & 12 & 10 & 57 & 10 & 20 & 10 & 8 \\
\hline & Gallinaza (Ga) ${ }^{2}$ & 150 & 25 & 100 & 0 & 0 & 122 & 20 & 82 & 0 & 0 \\
\hline & $\mathrm{Com}+\mathrm{Ga}^{3}$ & 220 & 37 & 125 & 12 & 10 & 180 & 30 & 102 & 10 & 8 \\
\hline \multirow{7}{*}{ ccN51 } & Testigo & 0 & 0 & 0 & 0 & 0 & 0 & 0 & 0 & 0 & 0 \\
\hline & $\mathrm{N}$ & 70 & 0 & 0 & 0 & 0 & 57 & 0 & 0 & 0 & 0 \\
\hline & $\mathrm{NP}$ & 70 & 12 & 0 & 0 & 0 & 57 & 10 & 0 & 0 & 0 \\
\hline & NPK & 70 & 12 & 25 & 0 & 0 & 57 & 10 & 20 & 0 & 0 \\
\hline & NPKS.Mg+Micros (Com) ${ }^{1}$ & 70 & 12 & 25 & 12 & 10 & 57 & 10 & 20 & 10 & 8 \\
\hline & Gallinaza (Ga) $)^{2}$ & 150 & 25 & 100 & 0 & 0 & 122 & 20 & 82 & 0 & 0 \\
\hline & $\mathrm{Com}+\mathrm{Ga}^{3}$ & 220 & 37 & 125 & 12 & 10 & 180 & 30 & 102 & 10 & 8 \\
\hline
\end{tabular}

Cuadro 1. Descripción de los tratamientos evaluados en la investigación.

\section{Resultados y Discusión}

En el Cuadro 2 se presentan las medias del efecto de los tratamientos de fertilización en el diámetro del tallo a los 360, 480, 600 y 720 días después del trasplante de los cultivares de cacao Nacional y CCN51. Dentro de cultivar y para cada uno de los tratamientos se observó una tendencia polinómica en el diámetro del tallo a medida que crece la planta. Se observa también que las diferencias en diámetro del tallo como efecto de los tratamientos de fertilización en cada uno de los cultivares fueron significativas $(P<$ 0.05) solamente a los 360 y 480 días. En promedio, no se observaron diferencias para diámetro del tallo $(\mathrm{P}<0.05)$ entre cultivares, a través de los tratamientos de fertilización, con una tendencia a mayor grosor en el cacao Nacional (Figura 1) lo cual podría ser un indicador de mayor capacidad productiva de los nuevos cacaos Nacionales (Ramos et al., 2001).

\begin{tabular}{|c|c|c|c|c|c|}
\hline \multirow[t]{2}{*}{ Genotipo } & \multirow[t]{2}{*}{ Fertilización } & \multicolumn{4}{|c|}{-.---- Días después del trasplante -..--..-- } \\
\hline & & 360 & 480 & 600 & 720 \\
\hline & & \multicolumn{4}{|c|}{...... Diámetro del tronco $(\mathrm{cm})^{*}$} \\
\hline \multirow{7}{*}{$\begin{array}{c}\text { Nacional } \\
5.39^{\mathrm{a}}\end{array}$} & Testigo & $2.7^{\mathrm{c}}$ & $4.1^{\mathrm{b}}$ & $5.0 \mathrm{a}$ & $5.9^{\mathrm{a}}$ \\
\hline & N & $3.8 \mathrm{ab}$ & $4.8^{\mathrm{ab}}$ & $6.0^{\mathrm{a}}$ & $6.8^{\mathrm{a}}$ \\
\hline & $\mathrm{NP}$ & $4.2^{\mathrm{a}}$ & $5.8^{\mathrm{a}}$ & $7.2^{\mathrm{a}}$ & $8.3^{\mathrm{a}}$ \\
\hline & NPK & $4.0 \mathrm{ab}$ & $5.6^{\mathrm{b}}$ & $6.0 \mathrm{a}$ & $6.1^{\mathrm{a}}$ \\
\hline & NPKSNIg+Micros (Com) & 3.7 abc & $5.4^{\text {ab }}$ & $6.3^{\mathrm{a}}$ & $7.2^{\mathrm{a}}$ \\
\hline & Gallinaza (Ga) $)^{2}$ & $3.1 \mathrm{bc}$ & $4.6 \mathrm{ab}$ & $5.7^{\mathrm{a}}$ & $6.3^{\mathrm{a}}$ \\
\hline & $\mathrm{Com}+\mathrm{Ga}^{3}$ & $3.9 \mathrm{ab}$ & $5.3^{\mathrm{ab}}$ & $6.4^{\mathrm{a}}$ & $7.3^{\mathrm{a}}$ \\
\hline \multirow{7}{*}{$\begin{array}{l}\text { CCN51 } \\
5.06 \mathrm{a}\end{array}$} & Testigo & $2.4^{\mathrm{b}}$ & $3.9^{\mathrm{b}}$ & $5.0^{\mathrm{a}}$ & $5.6^{\mathrm{a}}$ \\
\hline & $\mathrm{N}$ & $3.3 \mathrm{ab}$ & $4.8 \mathrm{ab}$ & $5.5^{\mathrm{a}}$ & $6.0^{\mathrm{a}}$ \\
\hline & $\mathrm{NP}$ & $3.8^{\mathrm{a}}$ & $5.4^{\text {ab }}$ & $6.2^{\mathrm{a}}$ & $6.8^{\mathrm{a}}$ \\
\hline & NPK & $3.7^{\mathrm{a}}$ & $5.5^{\mathrm{a}}$ & $6.2^{\mathrm{a}}$ & $7.0^{\mathrm{a}}$ \\
\hline & NPKSMg+Micros (Com) ${ }^{1}$ & $3.6^{\mathrm{a}}$ & $5.2 \mathrm{ab}$ & $6.1^{\mathrm{a}}$ & $6.8^{\mathrm{a}}$ \\
\hline & Gallinaza (Ga) ${ }^{2}$ & $3.2 \mathrm{ab}$ & $4.2 \mathrm{ab}$ & $5.2^{\mathrm{a}}$ & $6.2^{\mathrm{a}}$ \\
\hline & $\mathrm{Com}+\mathrm{Ga}^{3}$ & $3.2 \mathrm{ab}$ & $4.8^{\mathrm{ab}}$ & $6.0^{\mathrm{a}}$ & $6.3^{\mathrm{a}}$ \\
\hline
\end{tabular}

${ }^{*}$ Promedios en cada columna con letras iguales, dentro de genotipo, no difieren estadísticamente (Tukey $\mathrm{P}<0.05$ ) 'Tratamiento completo + micronutrientes; ${ }^{2}$ Gallinaza $\left(10 \mathrm{~kg}\right.$ planta $\left.{ }^{-1}\right) ;{ }^{3}$ Tratamiento completo + gallinaza

Cuadro 2. Diámetro de tallo para cacao nacional y clon $\mathrm{CCN}-5$ bajo diferentes tratamientos de fertilización 


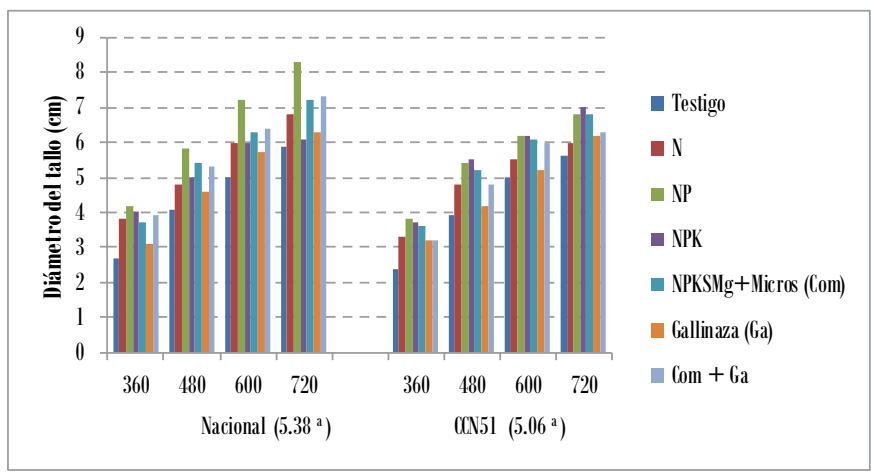

Figura 1. Efecto de los tratamientos de fertilización en el diámetro del tallo de dos cultivares de cacao a los 360, 480, 600 y 720 días después del trasplante.

Las medias del efecto de los tratamientos de fertilización en la altura de planta se presentan en el Cuadro 3. Se observa que las medias solamente fueron significativas $(P<0.05)$ a los 360 días, en ambos cultivares. Por otro lado, las medias de altura de planta de los cultivares, a través de los tratamientos de fertilización, fueron estadísticamente diferentes $(P<0.05)$ observándose que el cacao Nacional muestra una mayor altura $(\mathrm{P}<0.05)$ que el $\mathrm{CCN} 51$ (Figura 2).

\begin{tabular}{|c|c|c|c|c|c|}
\hline \multirow[t]{2}{*}{ Genotipo } & \multirow[t]{2}{*}{ Fertilización } & \multicolumn{4}{|c|}{--- Días después del trasplante -.--..- } \\
\hline & & 360 & 480 & 600 & 720 \\
\hline \multirow{8}{*}{$\begin{array}{c}\text { Nacional } \\
2.0 \mathrm{a}\end{array}$} & & \multicolumn{4}{|c|}{ Altura de la planta $(\mathrm{cm})^{*}$} \\
\hline & Testigo & $1.0^{b}$ & $1.7^{\text {a }}$ & $2.3^{\mathrm{a}}$ & $2.6^{\mathrm{a}}$ \\
\hline & n & $1.3^{\mathrm{a}}$ & $1.9 \mathrm{a}$ & $2.3^{\mathrm{a}}$ & $2.9 \mathrm{a}$ \\
\hline & $\mathrm{NP}$ & $1.3^{\mathrm{a}}$ & $1.9^{\mathrm{a}}$ & $2.3^{\mathrm{a}}$ & $2.8^{\mathrm{a}}$ \\
\hline & NPK & $1.3^{\mathrm{a}}$ & $1.8^{\mathrm{a}}$ & $2.3^{\mathrm{a}}$ & $2.8^{\mathrm{a}}$ \\
\hline & NPKSNIg+Micros (Com) ${ }^{1}$ & $1.3^{\mathrm{a}}$ & $1.8^{\mathrm{a}}$ & $2.3^{\mathrm{a}}$ & $2.9^{\mathrm{a}}$ \\
\hline & Gallinaza (Ga) ${ }^{2}$ & $1.2^{\mathrm{a}}$ & $1.7^{\mathrm{a}}$ & $2.1^{\mathrm{a}}$ & $2.5^{\mathrm{a}}$ \\
\hline & $\mathrm{Com}+6 \mathrm{a}^{3}$ & $1.3^{\mathrm{a}}$ & $1.8^{\mathrm{a}}$ & $2.2^{\mathrm{a}}$ & $2.7 \mathrm{a}$ \\
\hline \multirow{7}{*}{$\begin{array}{l}\text { CCN-51 } \\
1.80 \mathrm{~b}\end{array}$} & Testigo & $0.9 \mathrm{ab}$ & $1.4^{\mathrm{a}}$ & $2.0^{\mathrm{a}}$ & $2.5^{\mathrm{a}}$ \\
\hline & N & $1.1 \mathrm{ab}$ & $1.7^{\mathrm{a}}$ & $2.0^{\mathrm{a}}$ & $2.7^{\mathrm{a}}$ \\
\hline & $\mathrm{NP}$ & $1.2^{\mathrm{a}}$ & $1.7^{\text {a }}$ & $2.1^{\mathrm{a}}$ & $2.7 \mathrm{a}$ \\
\hline & NPK & $1.2^{\mathrm{a}}$ & $1.6^{\mathrm{a}}$ & $2.0^{\mathrm{a}}$ & $2.5 \mathrm{a}$ \\
\hline & NPKSMg+Micros (Com) ${ }^{1}$ & $1.1 \mathrm{ab}$ & $1.7^{\mathrm{a}}$ & $2.0^{\mathrm{a}}$ & $2.5^{\mathrm{a}}$ \\
\hline & Gallinaza (Ga) ${ }^{2}$ & $0.9^{b}$ & $1.4^{\mathrm{a}}$ & $2.0^{\mathrm{a}}$ & $2.5 \mathrm{a}$ \\
\hline & $\mathrm{Com}+\mathrm{Ga}^{3}$ & $1.1 \mathrm{ab}$ & $1.6^{\mathrm{a}}$ & $2.0^{\mathrm{a}}$ & $2.6^{\mathrm{a}}$ \\
\hline
\end{tabular}

ITratamiento completo + micronutrientes; ${ }^{2}$ Callinaza (10 kg planta-1); ${ }^{3}$ Tratamiento completo + gallinaza

Cuadro 3. Altura de la planta para cacao Nacional y clon CCN51 bajo diferentes tratamientos de fertilización.

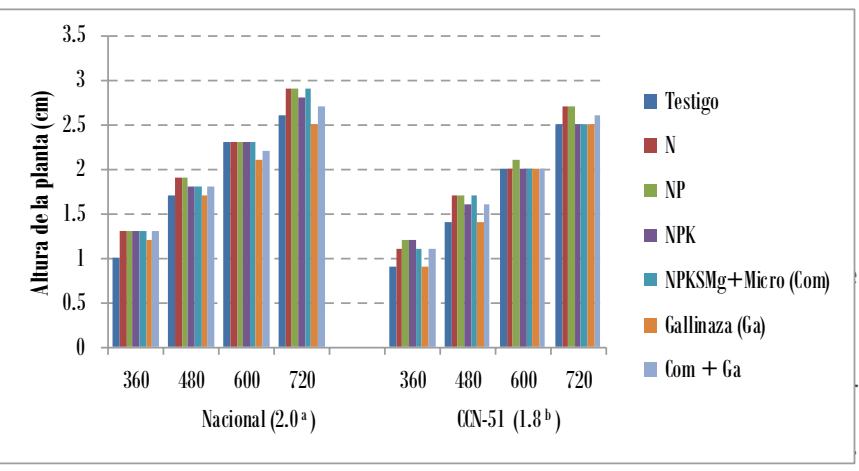

El índice vigor indica la habilidad de la planta para acumular biomasa y tendría efecto en la futura acumulación de rendimiento. Al igual que con las variables anteriores, el índice de vigor se incrementó con el transcurso del tiempo en cada uno de los tratamientos individuales de fertilización en los dos cultivares. No se observaron diferencias ( $P>0.05)$ entre las medias de los tratamientos de fertilización en cada uno de los cultivares. Al comparar las medias de los dos cultivares, a través de fertilización, se detectaron diferencias significativas $(P<0.05)$ confirmando la tendencia de mayor índice de vigor de los cultivares Nacionales (Figura 3). Además, el comportamiento en diámetro del tallo, altura e índice de vigor ratifica lo afirmado en la literatura (APROCAFA, 2004; Enríquez, 2004; Espinosa et al., 2006) que indica que el clon CCN51 es un cultivar de crecimiento erecto y de baja altura, condición que facilita y abarata las labores agronómicas, en comparación con los cultivares Nacionales que son mayor tamaño. 


\begin{tabular}{|c|c|c|c|c|}
\hline \multirow[t]{2}{*}{ Genotipo } & \multirow[t]{2}{*}{ Fertilización } & \multicolumn{3}{|c|}{-.-- Días después del trasplante -..-.... } \\
\hline & & 480 & 600 & 720 \\
\hline & & \multicolumn{3}{|c|}{ Indice de vigor $\left(\mathrm{m}^{3}\right)^{*}$} \\
\hline \multirow{7}{*}{$\begin{array}{c}\text { Nacional } \\
281.7^{\mathrm{a}}\end{array}$} & Testigo & $84.8^{\mathrm{a}}$ & $202.3^{\mathrm{a}}$ & $322.6^{\mathrm{a}}$ \\
\hline & N & $142.8^{\mathrm{a}}$ & $251.5^{\mathrm{a}}$ & $4489^{\mathrm{a}}$ \\
\hline & $\mathrm{NP}$ & $198.9^{\mathrm{a}}$ & $330.2^{\mathrm{a}}$ & $576.8^{\mathrm{a}}$ \\
\hline & NPK & $149.1^{\mathrm{a}}$ & $250.3^{\mathrm{a}}$ & $433.7^{\mathrm{a}}$ \\
\hline & NPKSMg+Micros (Com)1 & $167.2^{\mathrm{a}}$ & $288.6^{\mathrm{a}}$ & $462.1^{\mathrm{a}}$ \\
\hline & Gallinaza (Ga)2 & $111.8^{\mathrm{a}}$ & $206.5^{\mathrm{a}}$ & $343.5^{\mathrm{a}}$ \\
\hline & $\mathrm{Com}+\mathrm{Ga3}$ & $164.3^{\mathrm{a}}$ & $283.2^{\mathrm{a}}$ & $496.2^{\mathrm{a}}$ \\
\hline \multirow{7}{*}{$\begin{array}{l}C \mathrm{CN}-51 \\
227.0^{\mathrm{b}}\end{array}$} & Testigo & $66.7^{\mathrm{a}}$ & $137.2^{\mathrm{a}}$ & $253.5^{\mathrm{a}}$ \\
\hline & $\mathrm{N}$ & $114.5^{\mathrm{a}}$ & $186.6^{\mathrm{a}}$ & $312.2^{2}$ \\
\hline & NP & $137.3^{\mathrm{a}}$ & $249.7^{\mathrm{a}}$ & $434.8^{\mathrm{a}}$ \\
\hline & NPK & $153.5^{\mathrm{a}}$ & $235.6^{\mathrm{a}}$ & $416.4^{\mathrm{a}}$ \\
\hline & NPKSMg+Micros (Com) & $146.1^{\mathrm{a}}$ & $234.5^{\mathrm{a}}$ & $375.6^{\mathrm{a}}$ \\
\hline & Gallinaza (Ga) ${ }^{2}$ & $86.3^{\mathrm{a}}$ & $172.1^{\mathrm{a}}$ & $288.8^{\mathrm{a}}-\mathrm{C}-\mathrm{c}$ \\
\hline & $\mathrm{Com}+\mathrm{Ca}^{3}$ & $116.9^{\mathrm{a}}$ & $269.5^{\mathrm{a}}$ & $380.0^{\mathrm{a}}$ \\
\hline
\end{tabular}

* Promedios en cada columna con letras iguales, dentro de genotipo, no difieren estadísticamente (Tukey $\mathrm{P}<0.05$ ) 'Tratamiento completo + micronutrientes; ${ }^{2}$ Gallinaza (10 kg planta $\left.{ }^{-1}\right) ;{ }^{3}$ Tratamiento completo + gallinaza

Cuadro 4. Índice de vigor para cacao Nacional y clon $\mathrm{CCN}_{51}$ bajo diferentes tratamientos de fertilización.

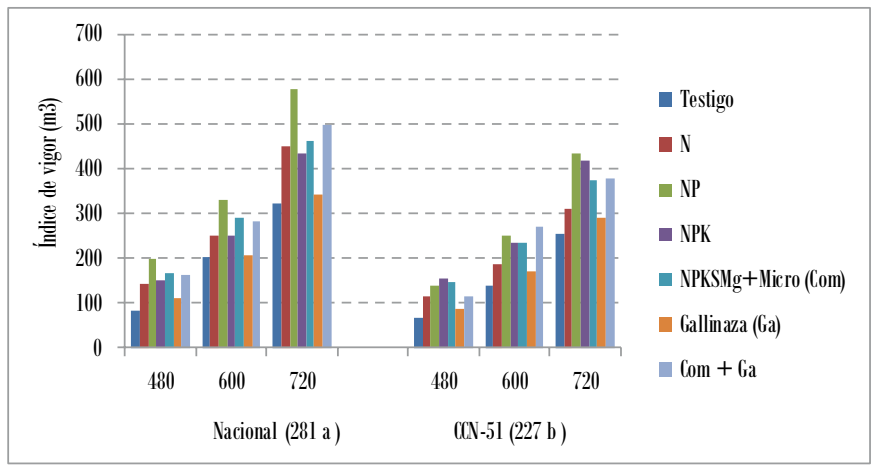

Figura 3. Efecto de los tratamientos de fertilización en el índice de vigor de la planta de dos cultivares de cacao a los 480.600 y 720 días después del trasplante.

El efecto de la fertilización en la incidencia de escoba de bruja se presenta en la Figura 4. Los datos se transformaron usando la expresión - (1/Y) para normalizarlos y proceder al análisis estadístico, las medias se presentan en la escala original para su discusión. No se encontraron diferencias estadísticas ( $P>0.05)$ entre la medias de los tratamientos de fertilización en cada uno de los cultivares evaluados y entre genotipos a través de los tratamientos de fertilización. La incidencia de escoba de bruja en las plantas de cacao durante el establecimiento del huerto experimental fue baja, confirmando lo reportado por APROCAFA (2004) que indica que que la escoba de bruja no representa un problema mayor durante el establecimiento de los materiales de cacao estudiados.

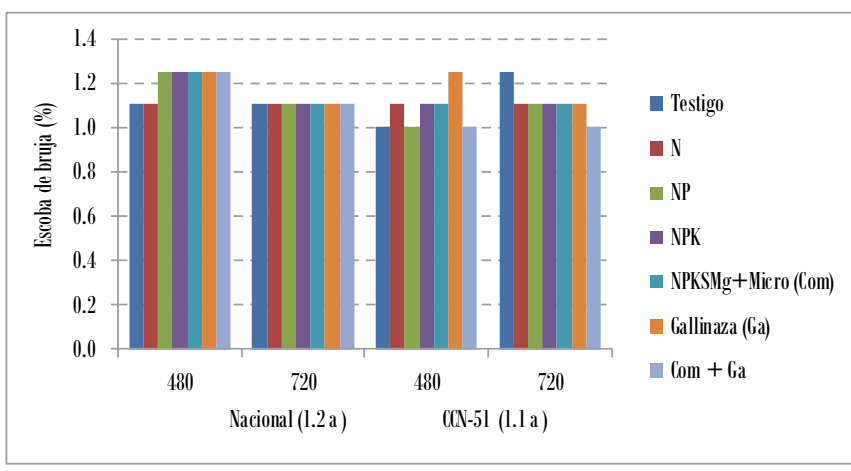

Figura 4. Efecto de los tratamientos de fertilización en la incidencia de escoba de bruja de dos cultivares de cacao a los 480.600 y 720 días después del trasplante.

Las plantas de cacao inician la producción de mazorcas luego de los dos primeros años del trasplante de las plántulas en el campo. A pesar de esto, la etapa de establecimiento de los cultivos perennes, como el cacao, es crucial para desarrollar en el tronco un sumidero de nutrientes y otros compuestos metabólicos que le permitan a la planta producir sin contratiempos en su edad adulta (Breure, 2012; Engels et al., 2012). Las plantaciones perennes que no han recibido una buena nutrición durante la etapa de establecimiento producen rendimientos inferiores a los que permite la oferta ambiental (Witt et al., 2005). Los productores de cacao generalmente descuidan la fase de establecimiento pensando que al no existir producción de mazorcas la planta no necesita una adecuada nutrición. La producción reportada en este experimento, colectada durante el segundo año después del trasplante, es marginal, pero pudiera ser un indicio del efecto de la nutrición en la futura producción de la huerta. Las medias del rendimiento de almendras secas se presentan en el Cuadro 5. Solamente se encontraron diferencias significativas $(P<0.05)$ para las medias de los tratamientos de fertilización en el cacao Nacional, pero se observó que las medias de rendimiento de los cultivares de cacao, a través de los tratamientos de fertilización, fueron significativas ( $P$ < 0.05). Los rendimientos del cultivar $\mathrm{CCN}_{51}$ fueron mayores que los del cacao tipo Nacional confirmando lo reportado por la literatura (APROCAFA, 2004; Espinosa et al., 2005). Aun cuando las diferencias entre tratamientos de fertilización en el cultivar $\mathrm{CCN}_{51}$ no fueron significativas, se observa una tendencia de mejor respuesta a la 
nutrición balanceada que la encontrada en el cacao Nacional (Figura

5). Este comportamiento sugiere que, a pesar de su menor tamaño, el sumidero de nutrientes y otros compuestos metabólicos se consolida tempranamente en el cultivar CCN51, condición que permitiría mayor acumulación de rendimiento en el periodo de producción de la edad adulta de la plantación.

\begin{tabular}{|c|c|c|}
\hline Genotipo & Fertilización & Rendimiento de almendras secas (kg/ha) \\
\hline \multirow{7}{*}{$\begin{array}{c}\text { Nacional } \\
203^{\mathrm{a}}\end{array}$} & Testigo & $102^{\mathrm{b}}$ \\
\hline & $\mathrm{N}$ & $130^{\mathrm{b}}$ \\
\hline & $\mathrm{NP}$ & $387^{\mathrm{a}}$ \\
\hline & NPK & $253^{\text {ah }}$ \\
\hline & NPKSMg+Micros (Com) $)^{1}$ & $194^{\text {at }}$ \\
\hline & Gallinaza (Ga) ${ }^{2}$ & $140^{\mathrm{b}}$ \\
\hline & $\mathrm{Com}+\mathrm{Ga}^{3}$ & $204^{\text {al }}$ \\
\hline \multirow{7}{*}{$\begin{array}{c}\text { CCN-51 } \\
316^{" ~}\end{array}$} & Testigo & $210^{\mathrm{a}}$ \\
\hline & $\mathrm{N}$ & $239^{\mathrm{a}}$ \\
\hline & $\mathrm{NP}$ & $344^{2}$ \\
\hline & NPK & $351^{\mathrm{a}}$ \\
\hline & NPKSHg+Micros (Com) ${ }^{1}$ & $335^{\mathrm{a}}$ \\
\hline & Gallinaza (Ga) ${ }^{2}$ & $428^{\mathrm{a}}$ \\
\hline & $\mathrm{Com}+\mathrm{Ga}^{3}$ & $306^{\mathrm{a}}$ \\
\hline \multicolumn{3}{|c|}{ * Promedios en cada columna con letras iguales, dentro de genotipo, no difieren estadísticamente (Tukey $\mathrm{P}<0.05$ ) } \\
\hline \multicolumn{3}{|c|}{ 'Tratamiento completo + micronutrientes; 'Gallinaza (10 kg planta $\left.{ }^{-1}\right)$; $^{3}$ Tratamiento completo + gallinaza } \\
\hline
\end{tabular}

Cuadro 5. Rendimiento de almendras secas de cacao Nacional y CCN51 bajo diferentes tratamientos de fertilización al segundo año del establecimiento del huerto.

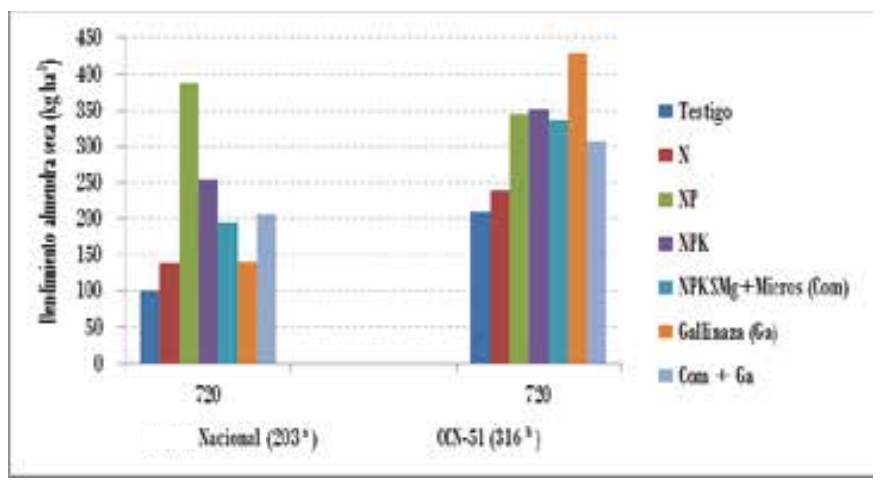

Figura 5. Efecto de los tratamientos de fertilización en el rendimiento de almendras secas al segundo año de establecimiento del huerto experimental.

\section{Conclusiones}

Los resultados de la evaluación de los dos años de establecimiento del huerto experimental de cacao mostraron diferencias en el crecimiento general de la planta. El cacao Nacional tiene más crecimiento que el CCN51 que tiende a ser más compacto. Esto ratifica lo encontrado por investigación conducida por otros investigadores en varios sitios en Ecuador. La incidencia de escoba de bruja fue baja en todos los tratamientos. El rendimiento del cacao CCN51 en el periodo de evaluación fue significativamente más alto que el del cacao Nacional lo que sugiere que este cultivar desarrolla el sumidero de nutrientes y otros compuestos temprano en el ciclo de crecimiento lo que aseguraría rendimientos más altos que los obtenidos con los materiales de cacao Nacionales.

\section{Bibliografía}

APROCAFA. 2004. Asociación de productores de cacao fino y de aroma. El gran cacao CCN-51 [En línea] www.sudnordnews. org/cgi-bin/ sudnordnews/ index.cgi (consultado el 25/05/2011).

CORPEI. 2009. Corporación de Promoción de Exportaciones e Inversiones [En línea] http://www.eluniverso. com/2009/10/17/1/1416/ cacaoteros (consultado el 21/03/2010).

Breure, K. 2012. Búsqueda de rendimiento en la palma de aceite: principios básico, p. 79-118, In T. Fairhurst and R. Hardter, eds. Palma de aceite: manejo para rendimientos altos y sostenibles, Primera ed. International Plant Nutrition Institute (IPNI) e International Potash Institute (IPI).

Engels, C., E. Kirkby, and P. White. 2012. Mineral nutrition, yield and source-sink relationships, p. 85-131, In P. Marschner, ed. Mineral Nutrition of Higher Plants, Third ed. Academic Press, London. 
Espinosa, J., F. Mite, S. Cedeño, S. Barriga, y J. Andino. 2006. Manejo por sitio específico del cacao basado en sistemas de Información Geográfica. Informaciones Agronómica 60:10-14.

Enríquez, G. 2004. Cacao orgánico. Instituto Nacional Autónomo de Investigaciones Agropecuarias, Quito, Ecuador.

INEC, 2012. Visualizador de estadísticas agropecuarias del Ecuador. [En línea] http://www.inec.gob.ec/estadisticas/ (consultado el 25/10/2012).

INIAP. 2009. Manual de cultivo de cacao para la Amazonía ecuatoriana. Instituto Nacional Autónomo de Investigaciones Agropecuarias (INIAP), Estación Experimental Central de la Amazonía. Coca, Ecuador.

López, R. 2007. Efecto de tres fitohormonas bajo diferentes dosis, sobre el desarrollo de palma africana (Elaeis guineensis Jacq.), en etapa de vivero, las golondrinas - Quinindé. 2006., Universidad Tecnológica Equinoccial, Santo Domingo, Ecuador.

Ramos, G.C. y Gómez A.M. 2001. Evolución de una plantanción de cacao Criollo Guarare. Relación entre la producción y parámetros de vigor. Agronomía Tropical 51(2): 175-186.

SICA. 2003. Censo Nacional Agropecuario. Resultados Nacionales y Provinciales. Ministerio de Agricultura y Ganadería. Ecuador.

Witt, C., T. Fairhurst, and W. Griffiths. 2005. The need to increase profitability in oil palm plantations: matching crop and nutrient management principles with evolving strategies. ISP National Seminar 5:1-22. 